\title{
以 $\mathrm{P} \mathrm{X}_{7}$ 受体为靶标的高通量篮选方法
}

\author{
蒋亚琴 $^{(12)}$ ，尹琪 ${ }^{(12)}$ ，栗世铀 ${ }^{(1 *}$ \\ (1) 中国科学院北京基因组研究所, 基因组科学及信息重点实验室, 北京 100029; \\ (2) 中国科学院大学, 北京 100049 \\ * 联系人, E-mail: lishiyou @ big.ac.cn \\ 2012-06-05 收稿, 2012-09-17 接受, 2013-06-14 网络版发表 \\ 中国科学院“百人计划”(2006-067)和国家自然科学基金(40872168)资助
}

\begin{abstract}
摘要 $\mathrm{P} 2 \mathrm{X}_{7}$ 受体是嘌呤-配体门控离子通道受体家族 $\mathrm{P} 2 \mathrm{X}$ 的一员. 一些研究表明, P2X 7 受 体主要在上皮细胞和免疫系统细胞中表达, 参与免疫调节的过程, 如炎性细胞因子释放、 膜融合及细胞凋亡. P $2 X_{7}$ 受体是神经退行性疾病、类风湿关节炎、神经性疼痛药物䇥选的 重要靶标, 已成为大型制药公司新的研究热点. 构建表达人源 $\mathrm{P} 2 \mathrm{X}_{7}$ 受体 $\left(\mathrm{hP} 2 \mathrm{X}_{7} \mathrm{R}\right)$ 的人胚 肾细胞系 HEK-293, 利用荧光成像分析系统(FlexStation), 针对 P2X 7 受体特性, 比较和验 证了 3 种用于检测 $\mathrm{P} 2 \mathrm{X}_{7}$ 受体高表达细胞系药理学特征的高通量荧光检测方法, 分别是膜 电位检测法、溴化乙啶内吞检测法和钙内流检测法. 对 3 种不同方法的比较研究表明, 钙 内流检测法简便、快捷, 更适用于 $\mathrm{P} 2 \mathrm{X}_{7}$ 受体调节剂的低成本、高通量药物篮选.
\end{abstract}

关键词

人源 $\mathrm{P} 2 \mathrm{X}_{7}$ 受体 高通量药物節选 荧光成像分析系统 膜电位检测 澳化乙啶内吞检测 钙内流检测
P2X 7 受体属于配体门控通道 $\mathrm{P} 2 \mathrm{X}$ 受体家族, 属 胞外核苷酸受体, 是继烟碱受体家族和谷氨酸受体 家族后第 3 类配体门控离子通道, 配体是 ATP (adenosine-5'-triphosphate). 迄今为止已经从哺乳动 物细胞中克隆了 7 个 $\mathrm{P} 2 \mathrm{X}$ 亚单位, 按照分布、离子通 透特性及对 ATP 的亲和力不同, 命名为 $\mathrm{P} 2 \mathrm{X}_{1 \sim 7}$ 受 体 ${ }^{[1,2]} . \mathrm{P} \mathrm{X}_{7}$ 受体主要在上皮细胞和免疫系统细胞中 表达, 如淋巴细胞、肥大细胞和巨噬细胞, 参与免疫 调节的过程, 如炎性细胞因子释放、膜融合及细胞调 $亡^{[3 \sim 5]}$. 近年的研究表明, 改良后的 $\mathrm{P} 2 \mathrm{X}_{7}$ 受体拮抗剂 AZD9056 已进人治疗类风湿关节炎的二期临床实

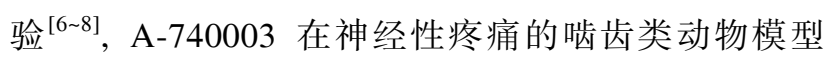
上表现出良好的药效 ${ }^{[9]} . \mathrm{P}^{2} \mathrm{X}_{7}$ 受体可能是治疗各种 神经退行性疾病、类风湿关节炎和神经性疼痛的靶 标 $^{[10 \sim 12]}$.

目前针对 $\mathrm{P} 2 \mathrm{X}_{7}$ 受体药理学特性的研究手段十分 有限. P2 $\mathrm{X}_{7}$ 受体在天然配体 ATP 或特异配体 Bz-ATP (3'-O-(4-benzoyl)-benzoyl adenosine-5'-triphosphate)
的短时间刺激下，形成非选择性阳离子通道，从而介 导细胞对钠离子、钾离子和钙离子等的通透性 ${ }^{[13]}$, 并 导致细胞膜去极化. 离子内流和膜电位的变化都可 用于建立 $\mathrm{P} 2 \mathrm{X}_{7}$ 受体的篮选检测方法.

电生理方法是公认的检测离子通道受体功能的 黄金标准，其中得到广泛应用的是全细胞膜片钳技 术. 这种方法灵敏、直接, 但操作复杂、成本昂贵, 无 法适用于高通量药物篮选. 最近几年来, 对膜电位变 化敏感的荧光染料也被广泛应用于离子通道受体的 功能检测. MD 公司(USA)的 FLIPR 系统适用的膜电 位检测试剂盒就是其中的代表，该方法在评估离子 通道活性及药理学功能方面与膜片钳数据具有很好 的相关性. 金属离子特异性的探针, 如钙离子指示剂, 也被用于检测细胞内金属离子的浓度变化. $\mathrm{P} 2 \mathrm{X}_{7}$ 受 体是可通过钙离子的阳离子通道, 当被激活剂激活 后, 细胞外的钙离子大量通过细胞膜进人细胞内, 从 而导致细胞内钲离子增加, 钙离子指示剂苂光强度 读数增强. $10.1007 / \mathrm{s} 11434-013-5870-7$ 
P2X 7 受体有一个和其他 P2X 亚型受体不同的特 性, 即在长时间高浓度的激活剂刺激下, 表达在细胞 膜表面的 $\mathrm{P}_{2} \mathrm{X}_{7}$ 受体不仅对阳离子通透, 而且选择性 的通道会形成孔洞，一些分子质量为几百道尔顿的 大分子也能自由通过细胞膜 ${ }^{[14,15]}$. 利用这一特性, 当 激活剂激活 $\mathrm{P} 2 \mathrm{X}_{7}$ 形成孔洞时, 分子质量为 $390 \mathrm{Da}$ 的 溴化乙啶进入细胞并与 DNA 相结合, 导致溴化乙啶 染料荧光强度的读数增强.

利用菼光成像分析系统(FlexStation), 针对人源 $\mathrm{P} 2 \mathrm{X}_{7}$ 受体特性, 构建得到了稳定表达 $\mathrm{P} 2 \mathrm{X}_{7}$ 受体的人 胚肾细胞系 HEK-293, 建立了 3 种用于检测 P2X 7 受 体高表达细胞系药理学特征的、高通量的荧光检测方 法一一膜电位检测法、澳化乙啶内吞检测法和钙内流 检测法. 通过比较 3 种不同的方法, 选定简便快捷的 钙内流检测法. 通过验证, 该模型稳定、灵敏, 适用 于高通量篮选 $\mathrm{P} 2 \mathrm{X}_{7}$ 受体调节剂, 为体外研究 $\mathrm{P} 2 \mathrm{X}_{7}$ 受体生理病理功能, 继而开发相关先导药物奠定了 基础.

\section{1 材料与方法}

(i ) 试剂. 所有细胞培养的相关培养基和试剂 购自 Gibco (Invitrogen, Carlsbad, CA). 小牛血清购自 Hyclone (ErembodegemAalst, Belgium). 细胞培养瓶、 细胞培养具、细胞培养板购自 Corning (Corning Inc, CA)和 BD 公司 (BD Clontech Palo Alto, CA). 96 孔及 384 孔板(黑壁透明底)购自 Costar (USA); $\mathrm{NaHCO}_{3}$ 等 常规试剂均为国产. 蓝色膜电位染料购自 Molecular Devices (Sunnyvale, CA). 免洗䥻离子检测试剂盒购 自 HD Biosciences (上海). 化学试剂 ATP, Bz-ATP 等 购自 Sigma-Aldrich (St. Louis, MO).

(ii) 重组表达质粒构建. 根据 NCBI 序列 (NCBI Accession No. NM_002562), 采用基因合成技 术获得人源 $\mathrm{P} 2 \mathrm{X}_{7}$ 受体基因, 并将其亚克隆到真核表 达载体 pCDNA3.1/hygro(+), 将构建所得重组表达质 粒命名为 $\mathrm{P} 2 \mathrm{X}_{7} \mathrm{R}-\mathrm{pCDNA} 3.1 / \mathrm{hygro}(+)$.

(iii) 稳定表达人源 $\mathrm{P} 2 \mathrm{X}_{7}$ 受体细胞系构建. 将 HEK-293 细胞培养在含有 $10 \%$ 小牛血清的 DMEM 培 养基中, 置于 $37^{\circ} \mathrm{C}, 5 \% \mathrm{CO}_{2}$ 温箱. 使用 DNA 转染试 剂 Lipofectamine ${ }^{\mathrm{TM}} 2000$ (Invitrogen, Carlsbad, CA), 将人源 $\mathrm{P} 2 \mathrm{X}_{7} \mathrm{R}$-pCDNA3.1/hygro(+)质粒转染人 HEK293 细胞, 形成 $H E K / h P 2 X_{7} R$ 重组细胞, 转染操作参 照试剂盒说明书, HEK-293 细胞为阴性对照. 利用
$100 \mu \mathrm{g} / \mathrm{mL}$ hygromycin (潮霉素), 对转染后的细胞进 行抗生素篮选, 2 周后挑选合适的单细胞克隆团, 于 96 孔细胞培养板中扩大培养 1 2 周. 激动剂作用于 细胞后能够引起胞内钙流变化, 根据上述原理进行 阳性细胞克隆的选择. 选择激动剂作用前后具有明 显钻流信号变化的细胞克隆(设定为 P0 代), 传代培 养, 并进行功能验证.

(iv) 反转录 PCR 分析. 参照使用说明书, 利用 Trizol (Invitrogen, Carlsbad, CA), 提取 HEK/hP2 $\mathrm{X}_{7} \mathrm{R}$ 重组细胞总 RNA. 在反转录系统中, 加人 $2 \mu \mathrm{g}$ 总 RNA 和 $1 \mu \mathrm{g}$ 随机引物, $70^{\circ} \mathrm{C}$ 反应 $5 \mathrm{~min}$ 后, 加人 $5 \times \mathrm{PCR}$ 缓 冲液, $0.5 \mathrm{mmol} / \mathrm{L}$ dNTP (dATP, dGTP, dCTP 和 dTTP) 混合物, 1 单位 RNase 抑制剂, $42^{\circ} \mathrm{C}$ 反应 $5 \mathrm{~min}$. 加人 200 单位 SuperScript II 反转录酶 (Life Technologies, Carlsbad, CA), $37^{\circ} \mathrm{C}$ 反应 $60 \mathrm{~min}, 70^{\circ} \mathrm{C}$ 加热 $5 \mathrm{~min}$ 使反 转录酶失活, 终止反应. 设计人源 $\mathrm{P} 2 \mathrm{X}_{7}$ 受体特异性 序列上游引物 5'-TCACTGCCGTCCCAAATACAGTTTCCG-3' (位于基因 801 827 位)和下游引物 5'TGCTGGTTCACCATCCTAATGTGGG-3' (位于基因 1220 1244 位).

（v）电生理记录. 常温下进行全细胞膜片钳记 录, 所用仪器为 EPC10 膜片钳放大器和数据采集器 (HEKA, Lambrecht, Germany), 记录电极电阻为 5 7 $\mathrm{M} \Omega$. 所充内液成分为 $\mathrm{CsCl} 154 \mathrm{mmol} / \mathrm{L}$, EGTA 10 $\mathrm{mmol} / \mathrm{L}, \mathrm{HEPES} 5 \mathrm{mmol} / \mathrm{L}$. 低浓度二价离子细胞外 液成分为 $\mathrm{NaCl} 147 \mathrm{mmol} / \mathrm{L}, \mathrm{KCl} 2 \mathrm{mmol} / \mathrm{L}, \mathrm{CaCl}_{2} 0.3$ $\mathrm{mmol} / \mathrm{L}, \mathrm{HEPES} 10 \mathrm{mmol} / \mathrm{L}$, 葡萄糖 $12 \mathrm{mmol} / \mathrm{L}$. 激 动剂通过 8 道程控灌流系统(ALA Scientific Instruments, Farmingdale, NY)给药.

( vi) 膜电位检测. 膜电位检测实验用细胞板选 用 Costar 公司的黑壁透明底、多聚赖氨酸包被的 96 孔板. 消化细胞后, 按照 $4 \times 10^{4}$ 细胞/孔的密度, 接种 到 96 孔检测细胞板, 每孔 $100 \mu \mathrm{L}$. 置于 $5 \% \mathrm{CO}_{2}$ 细胞 培养箱, $37^{\circ} \mathrm{C}$ 培养 $24 \mathrm{~h}$. 第 2 天, 弃去细胞培养基, 用 HBSS 缓冲液洗涤细胞 1 2 次后, 每孔加人 $50 \mu \mathrm{L}$ HBSS 缓冲液. 然后每孔加人 $50 \mu \mathrm{L}$ 蓝色膜电位检测 染料. 将检测细胞板置于 $5 \% \mathrm{CO}_{2}$ 温箱, $37^{\circ} \mathrm{C}$ 避光培 养 $45 \mathrm{~min}$. 配制 $5 \times$ 测试浓度的激动剂于化合物板中, 控制 DMSO 终浓度不超过 $0.5 \%$. 利用 FlexStation 苂 光成像分析系统(激发光波长 $488 \mathrm{~nm}$, 发射光波长 $550 \mathrm{~nm}$ ) 进行检测, 检测苂光读数 $18 \mathrm{~s}$ 时, 将激动剂 从化合物板加人待测细胞中, 同时采集数据直至 $300 \mathrm{~s}$ 
以上.

(vii) 溴化乙啶内吞检测. 选用 Costar 公司的黑 壁透明底、多聚赖氨酸包被的 96 孔板作为苂光染料 内吞检测实验用细胞板. 消化细胞后, 按照 $2 \times 10^{4}$ 细 胞/的密度, 接种到 96 孔检测细胞板, 每孔 $100 \mu \mathrm{L}$. 置于 $5 \% \mathrm{CO}_{2}$ 细胞培养箱, $37^{\circ} \mathrm{C}$ 培养 $24 \mathrm{~h}$. 第 2 天, 弃 去细胞培养基, 用 $350 \mu \mathrm{L} \mathrm{P} 2 \mathrm{X}_{7}$ 受体缓冲液(蔗糖 280 $\mathrm{mmol} / \mathrm{L}, \mathrm{KCl} 5.6 \mathrm{mmol} / \mathrm{L}, \mathrm{CaCl}_{2} 0.5 \mathrm{mmol} / \mathrm{L}$, 葡萄糖 $10 \mathrm{mmol} / \mathrm{L}$, HEPES $10 \mathrm{mmol} / \mathrm{L}, N$-methyl- $D$-glucamine $5 \mathrm{mmol} / \mathrm{L} ; \mathrm{pH}$ 7.4)洗涤细胞 2 次. 然后, 每孔加人 100 $\mu \mathrm{L}$ 溴化乙啶内吞染料(由 $\mathrm{P} 2 \mathrm{X}_{7} \mathrm{R}$ 缓冲液配制, 含有激 动剂、终浓度为 $100 \mu \mathrm{mol} / \mathrm{L}$ 的溴化乙啶). 将检测细 胞板置于 $5 \% \mathrm{CO}_{2}$ 细胞培养箱, $37^{\circ} \mathrm{C}$ 避光培养 $10 \mathrm{~min}$. 吸出溴化乙啶内吞染料, 每孔重新加人 $100 \mu \mathrm{L}$ $\mathrm{P} 2 \mathrm{X}_{7} \mathrm{R}$ 缓冲液. 设置 FlexStation 苂光成像分析系统的 激发波长为 $510 \mathrm{~nm}$, 发射波长为 $610 \mathrm{~nm}$, 开始检测 苂光读数. 如果检测拮抗剂的活性, 则在洗涤完细胞 后, 每孔加人 $50 \mu \mathrm{L}$ 含有拮抗剂的 $\mathrm{P} 2 \mathrm{X}_{7} \mathrm{R}$ 缓冲液, 置 于 $5 \% \mathrm{CO}_{2}$ 细胞培养箱, $37^{\circ} \mathrm{C}$ 避光培养 $30 \mathrm{~min}$. 用 $\mathrm{P} 2 \mathrm{X}_{7} \mathrm{R}$ 缓冲液配制 $2 \times$ 溴化乙啶内吞染料( $2 \times$ 激动剂与 终浓度 $200 \mu \mathrm{moL} / \mathrm{L}$ 的溴化乙啶), 每孔加人 $50 \mu \mathrm{L} 2 \times$ 溴化乙啶内吞染料, 继续培养 $10 \mathrm{~min}$ 后, 吸出溴化 乙啶内吞染料, 每孔重新加人 $100 \mu \mathrm{L} \mathrm{P} 2 \mathrm{X}_{7} \mathrm{R}$ 缓冲液. 在 FlexStation 苂光成像分析系统激发波长 $510 \mathrm{~nm}$, 发射波长 $610 \mathrm{~nm}$ 设置下开始检测苂光读数.

(viii) 钻内流检测. 选用黑壁透明底、多聚赖氨 酸包被的 96 孔板作为钙内流检测实验用细胞板. 消 化细胞, 按照 $4 \times 10^{4}$ 细胞/孔的密度, 每孔 $100 \mu \mathrm{L}$ 接种 到 96 孔检测细胞板, 置于 $5 \% \mathrm{CO}_{2}$ 细胞培养箱, $37^{\circ} \mathrm{C}$ 培养 $24 \mathrm{~h}$. 将 $1 \mathrm{mmol} / \mathrm{L}$ probenecid (丙磺舒)和 $1 \times$ 钲离 子指示剂( $\mathrm{HD}$ Biosciences, 上海)溶于 $\mathrm{P} 2 \mathrm{X}_{7} \mathrm{R}$ 缓冲液 中, 即得到钙离子敏感苂光染料. 第 2 天, 弃细胞培 养基, 每孔加人 $100 \mu \mathrm{L} 1 \times$ 钻离子敏感苂光染料. 将检 测细胞板置于 $5 \% \mathrm{CO}_{2}$ 细胞培养箱, $37^{\circ} \mathrm{C}$ 避光培养至少 $60 \mathrm{~min}$. 配制 $5 \times$ 测试浓度的激动剂于化合物板中, 控 制 DMSO 的终浓度不超过 $0.5 \%$. 设置 FlexStation 菼 光成像分析系统激发波长 $485 \mathrm{~nm}$, 发射波长 $525 \mathrm{~nm}$, 检测苂光读数, $18 \mathrm{~s}$ 时, 将激动剂从化合物板加入待 测细胞中, 同时采集数据直至 $120 \mathrm{~s}$. 而拮抗剂的活 性检测是通过二次加样的方式实现的, 先在细胞中 加人 $10 \mu \mathrm{L} 10 \times$ 拮抗剂, 避光孵育 $10 \mathrm{~min}$ 后开始检测 苂光值, $18 \mathrm{~s}$ 时, 将激动剂加人细胞, 并采集数据直
至 $120 \mathrm{~s}$.

(ix) 数据分析. 本实验中的苂光信号均由软件 SoftMax Pro (version 4.8, molecular devices)进行采集 分析. 使用软件 GraphPad Prism 4 对调节剂物剂量依 赖关系进行非线性曲线拟合分析. 本实验中的数据 都是经过 2 次重复实验所获得的平均值, 并且检测平 均标准偏差 $( \pm S E M) . Z$ 因子用以下公式计算 ${ }^{[16]}$

$$
Z=1-3 x\left(\left|S D_{\text {positive }}\right|+\left|S D_{\text {negtive }}\right|\right) /\left(\left|\mathrm{Mean}_{\text {positive }}\right|-\left|\mathrm{Mean}_{\text {negative }}\right|\right) \text {. }
$$

\section{2 结果与讨论}

\section{1 反转录 PCR 鉴定人源 $\mathrm{P}^{2} \mathrm{X}_{7}$ 受体的 RNA 表达}

为检测在得到的稳定细胞系中人源 $\mathrm{P} 2 \mathrm{X}_{7}$ 受体 mRNA 是否持续转录, 抽提出 $\mathrm{HEK} / \mathrm{hP} 2 \mathrm{X}_{7} \mathrm{R}$ 重组细胞 系及未经转染的 HEK-293 细胞的总 RNA, 通过反转 录 PCR 的方法进行了验证. 设计的扩增引物能扩增 得到人源 $\mathrm{P} 2 \mathrm{X}_{7}$ 受体基因 801 1244 位共计 $460 \mathrm{bp}$ 的 一段片段, 而且这个 $460 \mathrm{bp}$ 的片段中含有一个 $B a m \mathrm{H}$ I 酶切位点.

在以人源 $\mathrm{P} 2 \mathrm{X}_{7}$ 受体特异性序列为引物的反应中, 只有以 $\mathrm{HEK} / \mathrm{hP} 2 \mathrm{X}_{7} \mathrm{R}$ 稳定细胞系 RNA 为模板的体系 里产生了 $\mathrm{P} 2 \mathrm{X}_{7}$ 受体特异性条带 (460 bp) 而 HEK-293 母细胞系 RNA 中没有该扩增产物, 而且将该 $460 \mathrm{bp}$ 条带回收后, 用 $B a m \mathrm{H}$ I 酶切, 能够得到大小为 150 和 $310 \mathrm{bp}$ 的 2 条带(图 1), 这说明人源 $\mathrm{P} 2 \mathrm{X}_{7}$ 受体基因 能够在 $\mathrm{HEK} / \mathrm{hP} 2 \mathrm{X}_{7} \mathrm{R}$ 重组细胞中稳定表达, 适用于进 一步的细胞篮选.



图 1 RT-PCR 产物 2\% 琼脂糖电泳图

从 $\mathrm{HEK}-293$ 和 $\mathrm{HEK} / \mathrm{hP} 2 \mathrm{X}_{7} \mathrm{R}$ 细胞中分别提取制备 cDNA 作为 PCR 模板; 1 , HEK-293 细胞 cDNA 作为 PCR 模板的 PCR 产物; 2 , $\mathrm{HEK} / \mathrm{hP} 2 \mathrm{X}_{7} \mathrm{R}$ 细胞 cDNA 作为 PCR 模板的 PCR 产物; $3460 \mathrm{bp}$ $\mathrm{hP} 2 \mathrm{X}_{7} \mathrm{R} \mathrm{PCR}$ 产物经过 $B a m \mathrm{H}$ I 内切酶消化后的产物 


\section{$2.2 \mathrm{P} \mathrm{X}_{7}$ 受体的电生理特性}

表达有人源 $\mathrm{P} 2 \mathrm{X}_{7}$ 受体的 HEK-293 细胞上(钳制 电压-70 mV), 快速给予(1 3 s) Bz-ATP 刺激可以诱 发一个强度大约为 $5 \mathrm{nA}$ 的内向电流信号(图 2(a)). Bz-ATP 所诱发的内向电流呈剂量-效应相关, 随着剂 量的增加, 效应增强(图 2(c)). 图 2(b)显示了 HEK/ $\mathrm{hP} 2 \mathrm{X}_{7} \mathrm{R}$ 重组细胞对 Bz-ATP 的剂量-效应曲线. 拟合 曲线得出, $\mathrm{EC}_{50}$ 为 $110 \mu \mathrm{mol} / \mathrm{L}$. 在未转染人源 $\mathrm{P} 2 \mathrm{X}_{7}$ 受体的 HEK-293 细胞上, 大剂量的 Bz-ATP 刺激也无 法诱发内向电流. 由此可知, 所得到的 $\mathrm{HEK} / \mathrm{hP} 2 \mathrm{X}_{7} \mathrm{R}$ 重组细胞的细胞膜上有功能正常 $\mathrm{P} 2 \mathrm{X}_{7}$ 受体的表达.

\section{3 膜电位检测法鉴定 $\mathbf{P} 2 \mathrm{X}_{7}$ 受体功能}

当激活剂激活 $\mathrm{P} 2 \mathrm{X}_{7}$ 受体通道开启时, 阳性 $\mathrm{Na}^{+}$ 离子和 $\mathrm{Ca}^{2+}$ 离子进人并累积于细胞内, 细胞膜变为 去极化状态, 最终会导致膜电位染料菼光强度读数 增强. 运用该原理, 建立了 $\mathrm{HEK} / \mathrm{hP} 2 \mathrm{X}_{7} \mathrm{R}$ 细胞中人源 $\mathrm{P} 2 \mathrm{X}_{7}$ 受体通道功能的膜电位检测法. 加人人源 $\mathrm{P} 2 \mathrm{X}_{7}$
受体特异性激活剂 Bz-ATP 能检测到显著的膜电位染 料苂光读值的增加 (图 3). 分别用不同浓度的人源 $\mathrm{P} 2 \mathrm{X}_{7}$ 受体激活剂 Bz-ATP 及 ATP作用于 HEK/hP2 $\mathrm{X}_{7} \mathrm{R}$ 重组细胞系, 并检测细胞膜电位的变化. 实验结果显 示, 随着激活剂浓度的增加, 苂光钙信号不断增强, 表现出剂量依赖效应, Bz-ATP 的 $\mathrm{EC}_{50}$ 值为 27.3 $\mu \mathrm{mol} / \mathrm{L}, \mathrm{ATP}$ 的 $\mathrm{EC}_{50}$ 值为 $169.2 \mu \mathrm{mol} / \mathrm{L}$ (图 4).

\section{4 溴化乙啶内吞检测法鉴定 $\mathrm{P}_{2} \mathrm{X}_{7}$ 受体功能}

当高浓度激动剂长时间刺激 $\mathrm{P} 2 \mathrm{X}_{7}$ 受体时, 表达 在细胞膜表面的 $\mathrm{P} 2 \mathrm{X}_{7}$ 受体不仅对阳离子通透, 而且 选择性的通道会形成孔洞, $390 \mathrm{Da}$ 溴化乙啶进人细胞 并与 DNA 相结合, 导致溴化乙啶染料苂光强度读数 增强. 运用该原理, 建立了 $\mathrm{HEK} / \mathrm{hP} 2 \mathrm{X}_{7} \mathrm{R}$ 细胞中人源 $\mathrm{P} 2 \mathrm{X}_{7}$ 受体通道功能的溴化乙啶内吞检测法. 图 5 显 示的是细胞被不同激活剂刺激后, 苂光显微镜下所 观察到的苂光. 在未转染人源 $\mathrm{P} 2 \mathrm{X}_{7}$ 受体的 HEK-293 细胞上, $50 \mu \mathrm{mol} / \mathrm{L}$ ATP 刺激 $1 \mathrm{~h}$ 也观测不到任何的苂 光变化. (a)

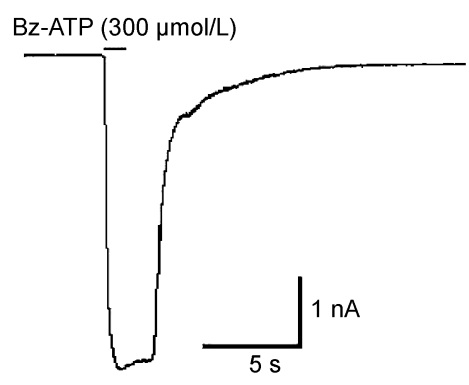

(b)

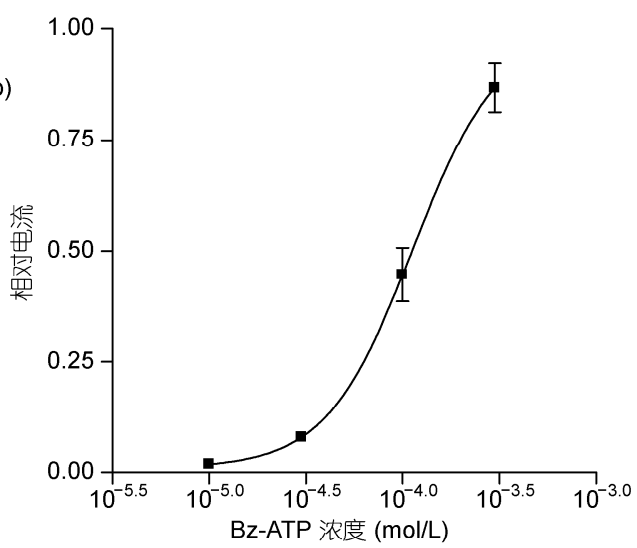

(c)

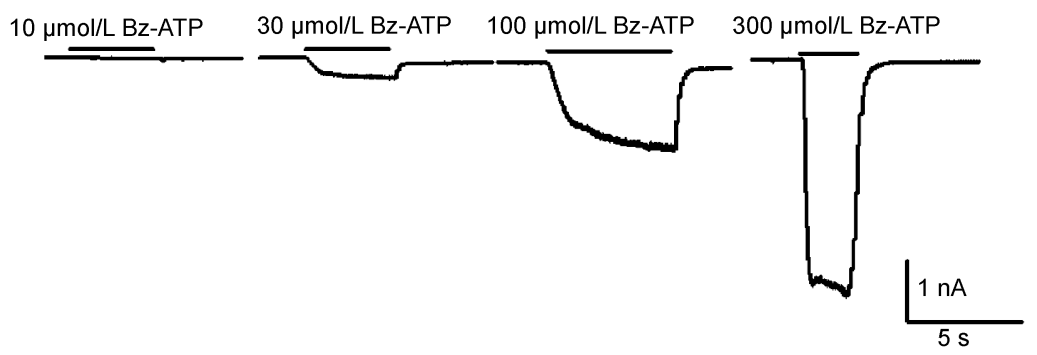

图 2 Bz-ATP 刺激 $\mathrm{HEK} / \mathrm{hP} 2 \mathrm{X}_{7} \mathrm{R}$ 细胞引发的内向电流信号

(a) 最高浓度 Bz-ATP 刺激 $\mathrm{HEK} / \mathrm{hP} 2 \mathrm{X}_{7} \mathrm{R}$ 重组细胞引发的内向电流信号, 缓冲液含有 $0.3 \mathrm{mmol} / \mathrm{L} \mathrm{CaCl}_{2}$, 不含镁离子(低二价离子缓冲液);

(b) 低二价离子缓冲外液环境下, HEK/hP2X $\mathrm{X}_{7} \mathrm{R}$ 重组细胞对 Bz-ATP 的剂量依赖曲线, 每个数据点重复 5 次实验; (c) 不同浓度 Bz-ATP 刺激 $\mathrm{HEK} / \mathrm{hP} 2 \mathrm{X}_{7} \mathrm{R}$ 细胞引发的内向电流信号 


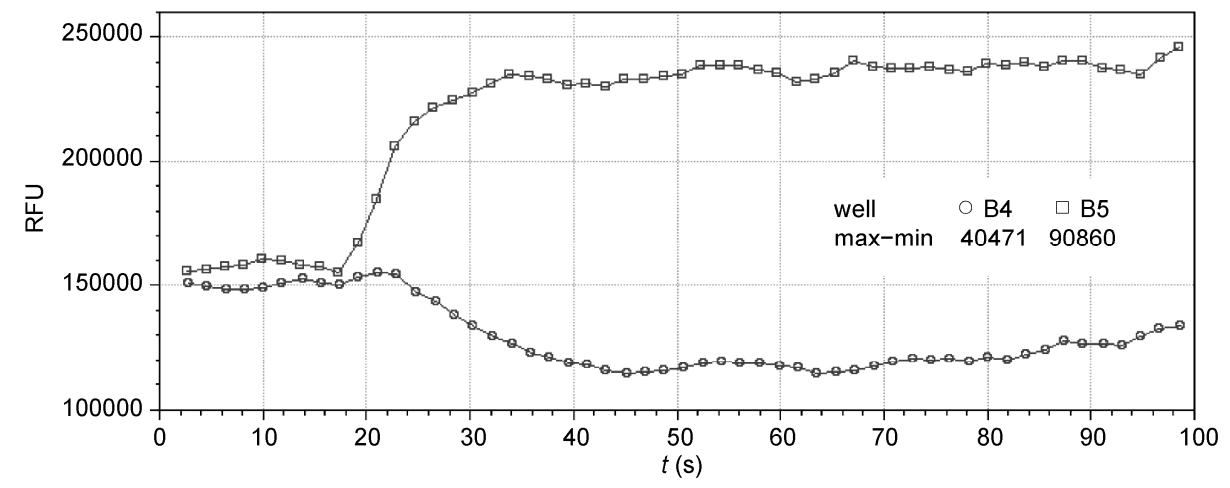

图 3 典型的膜电位染料在 FlexStation 读板仪上的荧光信号读值

“○”代表未经转染的 HEK-293 母细胞; “口”代表稳定表达 hP2X 7 受体的 HEK-293 细胞; well 代表孔; B4, B5 代表样品所在孔的位置; $\max -\min$ 代表最大信号值与最小信号值的差; 在 $16 \mathrm{~s}$ 时, 加人 $100 \mu \mathrm{mol} / \mathrm{L} \mathrm{Bz}-\mathrm{ATP}$ 刺激细胞


图 $4 \mathrm{hP2X}_{7}$ 受体重组细胞系对化合物的剂量依赖曲线

(a) $\mathrm{HEK} / \mathrm{hP} 2 \mathrm{X}_{7} \mathrm{R}$ 重组细胞系对 Bz-ATP 的剂量依赖曲线; (b) HEK/hP2 $\mathrm{X}_{7} \mathrm{R}$ 重组细胞系对 ATP 的剂量依赖曲线; $\mathrm{EC}_{50}$ 数值使用 $\mathrm{GraphPad}$ Prism 4 软件分析得到

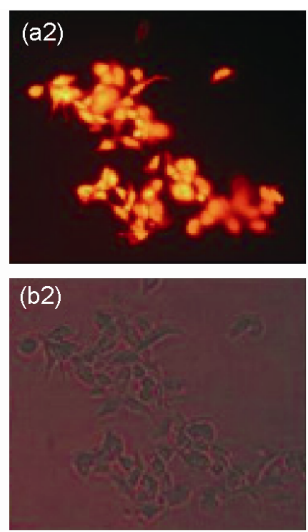
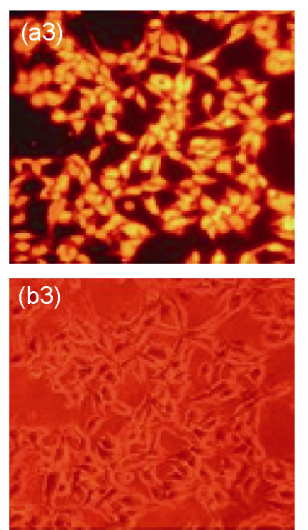
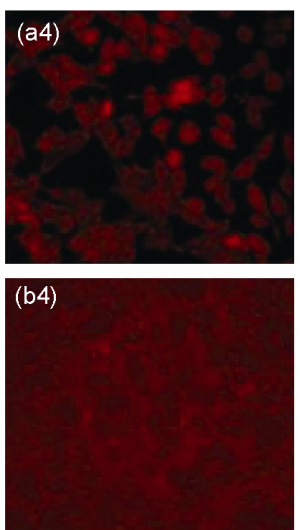

图 5 不同激动剂刺激 $\mathrm{HEK} / \mathrm{hP2X} \mathrm{X}_{7} \mathrm{R}$ 细胞引发的 EB 内吞荧光信号

(a) 苂光视野下的细胞; (b) 可见光视野下的细胞; 1, $10 \mu \mathrm{mol} / \mathrm{L}$ 2-Mes-ATP (2-methylthioadenosine triphosphate tetrasodium, 二甲基硫 ATP) 刺激 $\mathrm{HEK} / \mathrm{hP} 2 \mathrm{X}_{7} \mathrm{R}$ 重组细胞; $2,10 \mu \mathrm{mol} / \mathrm{L} \mathrm{Bz}-\mathrm{ATP}$ 刺激 HEK $/ \mathrm{hP} 2 \mathrm{X}_{7} \mathrm{R}$ 重组细胞; 3, $50 \mu \mathrm{mol} / \mathrm{L}$ ATP 刺激 HEK/hP2 $\mathrm{X}_{7} \mathrm{R}$ 重组细胞; 4 , 缓冲 液刺激 $\mathrm{HEK} / \mathrm{hP} 2 \mathrm{X}_{7} \mathrm{R}$ 重组细胞. 5, $50 \mu \mathrm{mol} / \mathrm{L}$ ATP 刺激 HEK-293 母细胞 
分别用不同浓度的 $\mathrm{P} 2 \mathrm{X}_{7}$ 受体激活剂 Bz-ATP, ATP 及 2-Mes-ATP 作用于 HEK/hP2 $\mathrm{X}_{7} \mathrm{R}$ 重组细胞系, 检测苂光染料 $\mathrm{EB}$ 的苂光读数变化. 结果显示, 随着 激活剂浓度增加, 苂光信号不断增强, 表现出剂量依 赖效应, Bz-ATP 的 $\mathrm{EC}_{50}$ 值为 $4.22 \mu \mathrm{mol} / \mathrm{L}$, ATP 的 $\mathrm{EC}_{50}$ 值为 $42.88 \mu \mathrm{mol} / \mathrm{L}, 2-\mathrm{Mes}-\mathrm{ATP}$ 的 $\mathrm{EC}_{50}$ 值为 84.45 $\mu \mathrm{mol} / \mathrm{L}$ (图 6). $\mathrm{P} 2 \mathrm{X}_{7}$ 受体特异性的拮抗剂异喹啉衍生 物 KN62 (1-[N,O-bis (1,5-isoquinolinesulphonyl)- $N$ methyl- $L$-tyrosyl]-4-phenylpiperazine) 对 $\mathrm{P}^{2} \mathrm{X}_{7}$ 受体的 抑制效果也通过荧光染料 $\mathrm{EB}$ 的内吞实验得到验证, $\mathrm{KN} 62$ 的 $\mathrm{IC}_{50}$ 值为 $466 \mathrm{nmol} / \mathrm{L}$ (图 6(d)).

\section{5 钻内流检测法鉴定 $\mathbf{P} 2 \mathrm{X}_{7}$ 受体功能}

表达在细胞膜表面的 $\mathrm{P} 2 \mathrm{X}_{7}$ 受体是阳离子选择性 离子通道, 被激活开放之后, $\mathrm{P} 2 \mathrm{X}_{7}$ 受体能介导一价和 二价的阳离子通透 $\left(\mathrm{Na}^{+}, \mathrm{K}^{+}, \mathrm{Ca}^{2+}\right.$ 等$)$. 运用该原理, 建立了 $\mathrm{HEK} / \mathrm{hP} 2 \mathrm{X}_{7} \mathrm{R}$ 细胞中人源 $\mathrm{P} 2 \mathrm{X}_{7}$ 受体通道功能 的钙内流检测法. 图 7 显示的是利用 HDB 公司的钙
离子指示剂，用 $\mathrm{Bz}-\mathrm{ATP}$ 激活 $\mathrm{P} 2 \mathrm{X}_{7}$ 受体，能够检测到 7 9 倍的苂光信号的增加.

分别用不同浓度的 $\mathrm{P} 2 \mathrm{X}_{7}$ 受体激活剂 Bz-ATP, ATP 及 2-Mes-ATP 作用于 HEK/hP2 $\mathrm{X}_{7} \mathrm{R}$ 重组细胞系, 检测由钲离子内流所引发的苂光读数的变化. 实验 结果显示, 随着激活剂浓度的增加, 苂光信号不断增 强, 表现出剂量依赖效应, Bz-ATP 的 $\mathrm{EC}_{50}$ 值为 10.54 $\mu \mathrm{mol} / \mathrm{L}, \mathrm{ATP}$ 的 $E C_{50}$ 值为 $50.8 \mu \mathrm{mol} / \mathrm{L}, 2$-MesATP 的 $\mathrm{EC}_{50}$ 值为 $55 \mu \mathrm{mol} / \mathrm{L}$ (图 8). $\mathrm{P} 2 \mathrm{X}_{7}$ 受体特异性的怙抗剂 $\mathrm{KN} 62$ 对 $\mathrm{P} 2 \mathrm{X}_{7}$ 受体的抑制效果也得到验证, KN62 的 $\mathrm{IC}_{50}$ 值为 $437 \mathrm{nmol} / \mathrm{L}$ (图 8(d)). 这一数据与前面的电 生理实验、膜电位检测实验及溴化乙啶内吞实验所得 到的数据在同一数量级别.

\section{6 钙内流检测法的 $Z$ 因子评估}

以上实验结果表明, 相对于其他 2 种方法, 钙离 子内流检测信号高、步骤简便, 更适宜应用于高通量 药物篎选. 为验证构建的 HEK/hP2 $\mathrm{X}_{7} \mathrm{R}$ 高通量篮选系
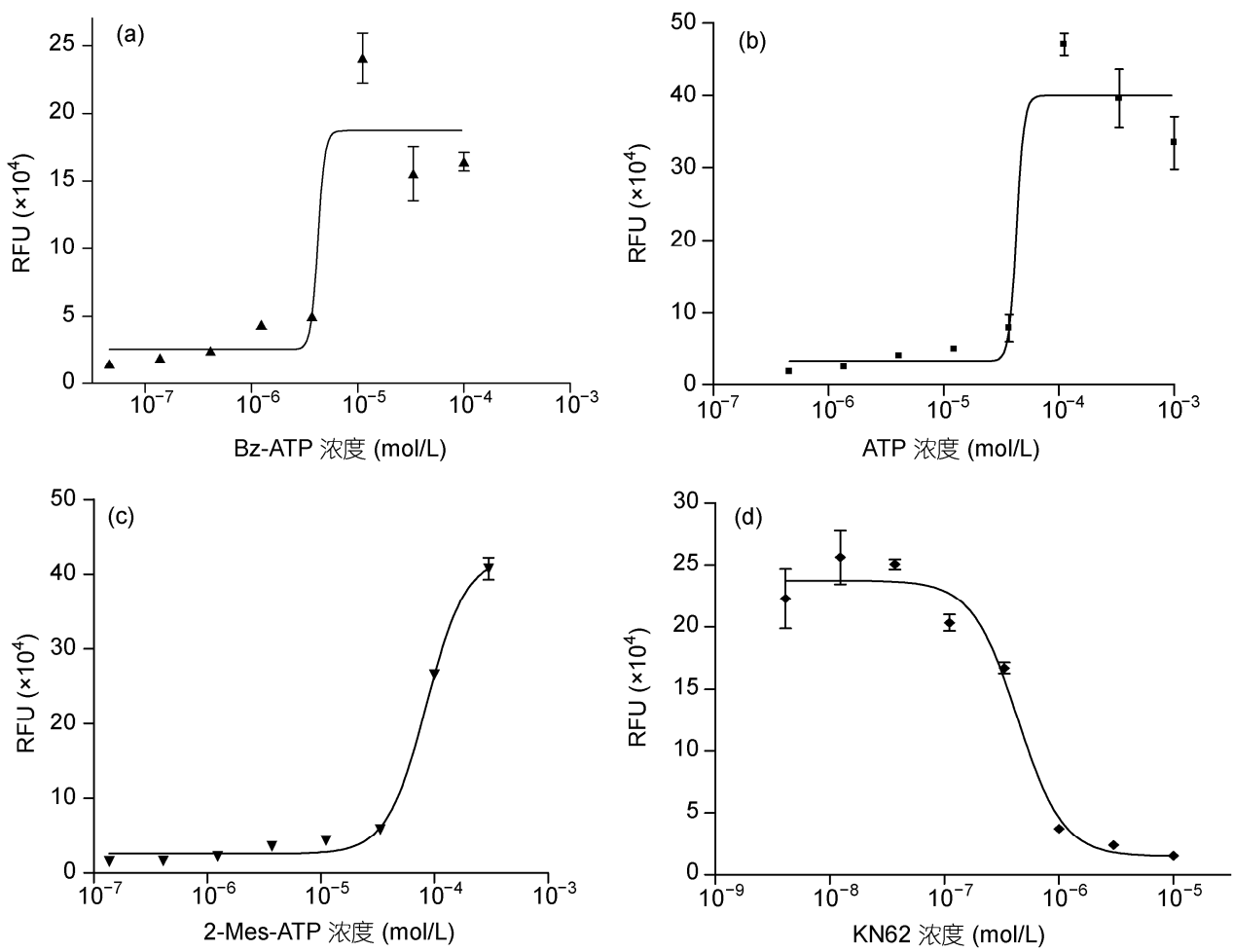

图 $6 \mathrm{HEK} / \mathrm{hP2} \mathrm{X}_{7} \mathrm{R}$ 重组细胞系对化合物的剂量依赖曲线

(a) HEK/hP2 $\mathrm{X}_{7} \mathrm{R}$ 重组细胞系对 Bz-ATP 的剂量依赖曲线; (b) HEK/hP2 $\mathrm{X}_{7} \mathrm{R}$ 重组细胞系对 ATP 的剂量依赖曲线; (c) HEK/hP2 $\mathrm{X}_{7} \mathrm{R}$ 重组细 胞系对 2-Mes-ATP 的剂量依赖曲线; (d) HEK/hP2 $\mathrm{X}_{7} \mathrm{R}$ 重组细胞系对 $\mathrm{KN} 62$ 的剂量依赖曲线; $\mathrm{EC}_{50}$ 或 $\mathrm{IC}_{50}$ 数值使用 $\mathrm{GraphPad}$ Prism 4 软件 分析得到 


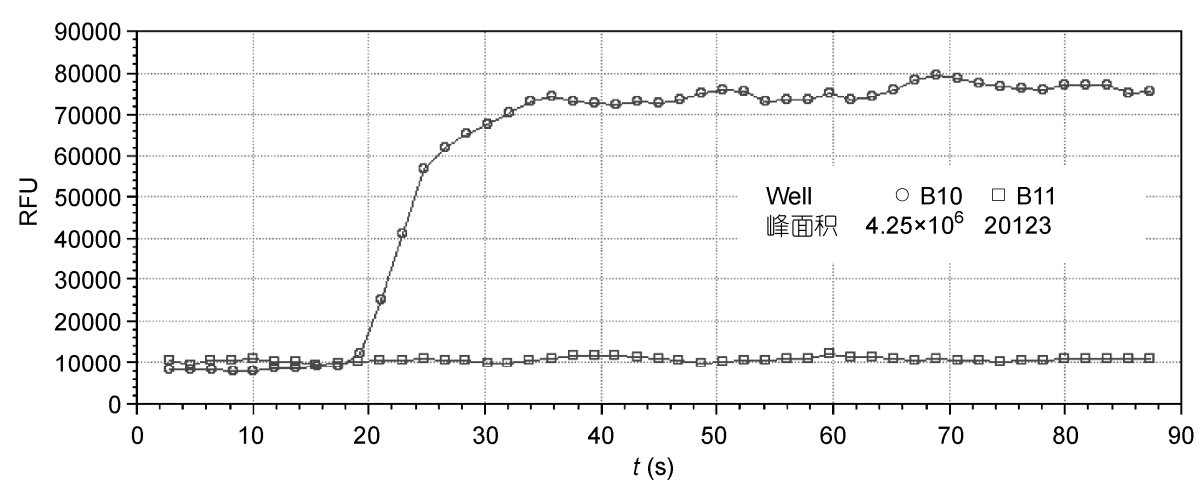

图 7 典型的钙离子指示剂在 FlexStation 读板仪上的荧光信号读值

“口”代表未经转染的 HEK-293 母细胞; “○”代表稳定表达 hP2X 7 受体的 HEK-293 细胞; well 代表孔; B10, B11 代表样品所在孔的位置; 在 $16 \mathrm{~s}$ 时, 加人 $100 \mu \mathrm{mol} / \mathrm{L} \mathrm{Bz}-\mathrm{ATP}$ 刺激细胞
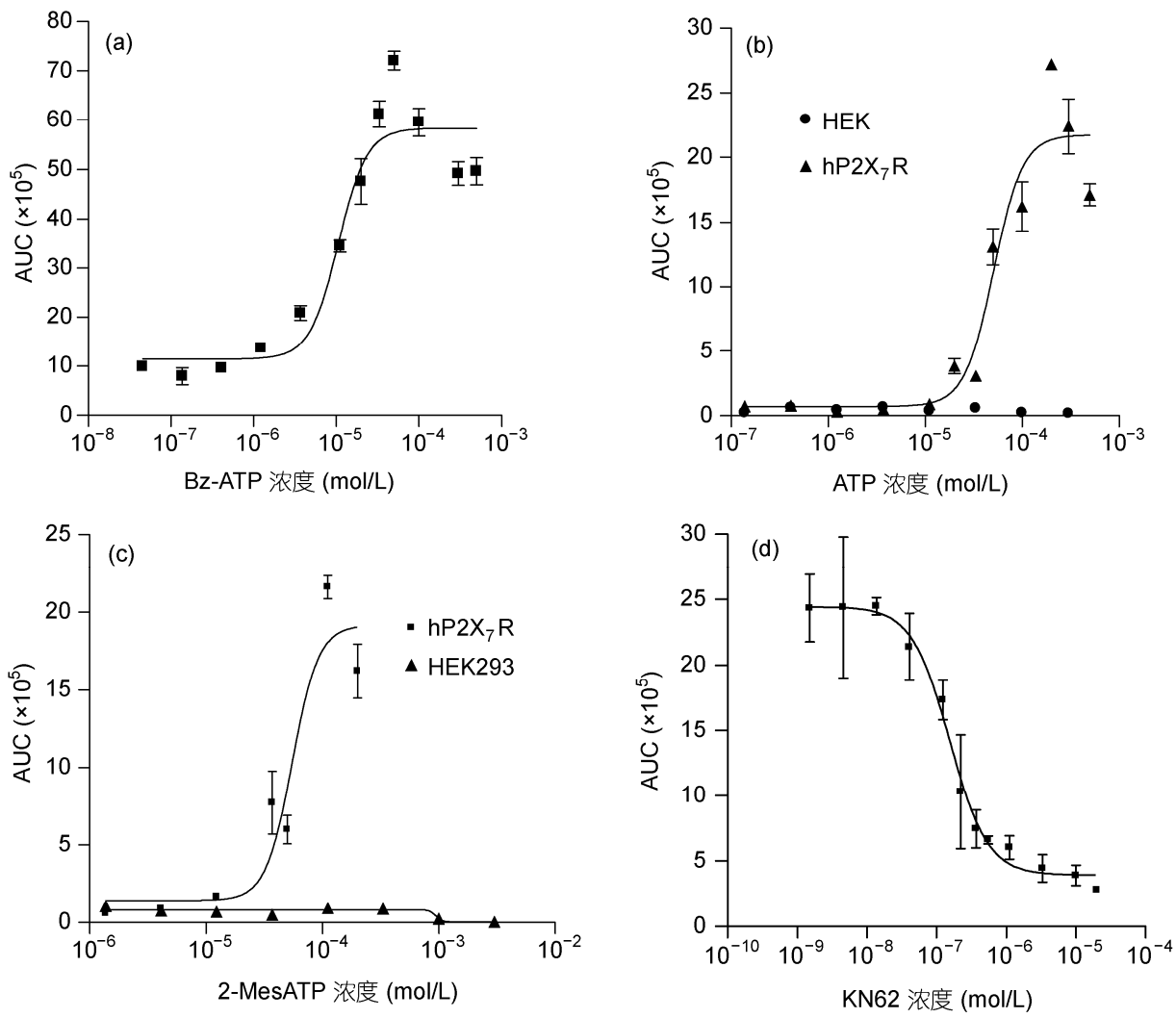

图 $8 \mathrm{HEK} / \mathrm{hP2X} \mathrm{X}_{7} \mathrm{R}$ 重组细胞系对化合物的剂量依赖曲线

(a) HEK/hP2 $X_{7} \mathrm{R}$ 重组细胞系对 Bz-ATP 的剂量依赖曲线; (b) HEK/hP2 $\mathrm{X}_{7} \mathrm{R}$ 重组细胞系对 ATP 的剂量依赖曲线; (c) HEK/hP2 $\mathrm{X}_{7} \mathrm{R}$ 重组细 胞系对 2-Mes-ATP 的剂量依赖曲线; (d) HEK/hP2 $\mathrm{X}_{7} \mathrm{R}$ 重组细胞系对 KN62 的剂量依赖曲线; $\mathrm{EC}_{50}$ 或 $\mathrm{IC}_{50}$ 数值使用 $\mathrm{GraphPad}$ Prism 4 软件 分析得到

统的稳定性和可靠性, 本研究采用了 $Z$ 因子法来评估. $Z$ 因子综合考虑了实验检测到的信号的变化范围和 波动范围, 反映了方法的信噪比及数据的偏差, 因此 可作为高通量笁选系统的衡量标准. $Z$ 因子与篮选源 无关，只与篮选方法本身有关，如果 $Z$ 因子高于 0.5 ,
说明篮选方法适用于高通量篮选; 如果低于 0.5 , 则 说明篮选方法不适用于高通量篮选，无法得到可靠 的数据, 尚需进一步优化.

首先检测了一整板 96 孔板的 $Z$ 因子值，将一整 块 96 孔板的 6 列加人 $30 \mu \mathrm{mol} / \mathrm{L} \mathrm{Bz}-\mathrm{ATP}$ (激动剂的 
$E C_{100}$ 浓度, 10 倍 $E C_{50}$ 浓度)作为阳性对照(positive), 同时另外 6 列加人缓冲液作为阴性对照 (negative), 各 孔中 DMSO 的终浓度均为 $0.2 \%$, 采集数据 $80 \mathrm{~s}$. 测试 结果 $Z$ 因子值为 0.796 , 信号窗口值为 19.36 (图 9).

在正常的高通量篮选实验中, 会一次性检测十 几甚至几十板 96 孔板, 为了检测篮选板与板间的差 异性, 连续测试了 10 块 96 孔板的 $Z$ 因子值(表 1). 每 块板随机选择 2 列进行测试, 其中 1 列加人 30 $\mu \mathrm{mol} / \mathrm{L} \mathrm{Bz}-\mathrm{ATP}$ (激动剂的 $\mathrm{EC}_{100}$ 浓度, 10 倍 $\mathrm{EC}_{50}$ 浓度) 作为阳性对照, 另 1 列加人缓冲液作为阴性对照, 各孔中 DMSO 的终浓度均为 $0.2 \%$, 结果见表 1 , 各次 检测 $Z$ 因子均大于 0.5 . 因此, 该 $\mathrm{HEK} / \mathrm{hP} 2 \mathrm{X}_{7} \mathrm{R}$ 篮选 模型适于应用 96 孔板进行 $\mathrm{P} 2 \mathrm{X}_{7}$ 受体调节剂的高通量 篮选.
在本文中，利用了多种 $\mathrm{P} 2 \mathrm{X}_{7}$ 受体作为离子通道 受体的特性，建立了多种检测方法. 其中有黄金标 准一一全细胞膜片钳法, 以及 3 种不同的苂光检测方 法一一利用膜电位敏感苂光染料的膜电位检测法, 利用钙离子敏感苂光染料的钙内流检测方法和利用 核酸敏感荧光染料的溴化乙啶内吞检测法. 在不同 的检测系统中，检测了激活剂和抑制剂的药理学 特性.

所使用的多种检测方法都能有效地测定人 $\mathrm{P} 2 \mathrm{X}_{7}$ 受体的功能, 但是每种方法各有优缺点. 全细胞膜片 钳法虽然是黄金标准，但是这种方法要求有专业的 操作人员, 实验通量低, 成本昂贵, 并不适应高通量 药物篮选的要求. 3 种不同的苂光检测方法实验通量 高，可以实现 96 孔板甚至 384 孔板的实验通量，相对

(a)
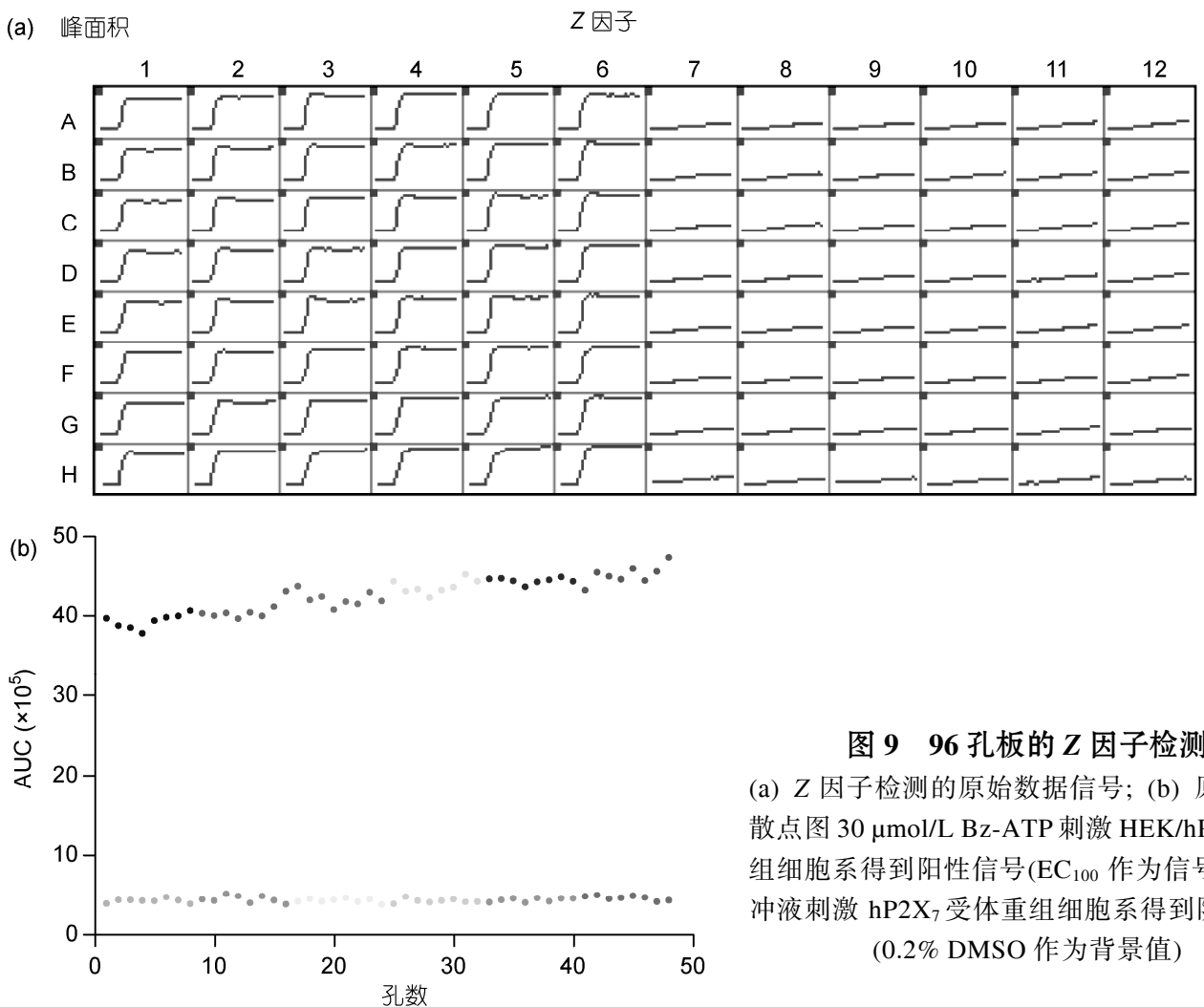

图 996 孔板的 $Z$ 因子检测

(a) $Z$ 因子检测的原始数据信号; (b) 原始数据 散点图 $30 \mu \mathrm{mol} / \mathrm{L} \mathrm{Bz}-\mathrm{ATP}$ 刺激 HEK/hP2X $\mathrm{X}_{7} \mathrm{R}$ 重 组细胞系得到阳性信号 $\left(\mathrm{EC}_{100}\right.$ 作为信号值), 缓 冲液刺激 $\mathrm{hP} \mathrm{X}_{7}$ 受体重组细胞系得到阴性信号 ( $0.2 \%$ DMSO 作为背景值)

表 110 块 96 孔板的 $Z$ 因子检测值 ${ }^{a)}$

\begin{tabular}{cccccccccccc}
\hline 板号 & 1 & 2 & 3 & 4 & 5 & 6 & 7 & 8 & 9 & 10 \\
\hline$Z$ 因子 & 0.834 & 0.898 & 0.874 & 0.861 & 0.908 & 0.882 & 0.831 & 0.866 & 0.793 & 0.870 \\
\hline
\end{tabular}

a) 将 $\mathrm{hP}_{2} \mathrm{X}_{7}$ 受体重组细胞接种于 10 块 96 孔板中, $30 \mu \mathrm{mol} / \mathrm{L}$ Bz-ATP 刺激 $\mathrm{HEK} / \mathrm{hP} 2 \mathrm{X}_{7}$ 受体重组细胞系得到阳性信号 $\left(\mathrm{EC}_{100}\right.$ 作为信 号值), 缓冲液刺激 $\mathrm{HEK} / \mathrm{hP} 2 \mathrm{X}_{7}$ 受体重组细胞系得到阴性信号, 计算每块板的 $Z$ 因子值 
来说成本低, 可以用于高通量药物篮选. 但是膜电位 检测法信噪比低, 信号窗口小, 而且 HEK-293 母细 胞有一定量的背景值; 溴化乙啶内吞检测法实验步 骤繁琐, 需要多次清洗细胞孔板, 这都大大影响到了
这 2 种方法的大规模笁选可操作性. 相比较而言, 钙 内流检测方法有着 10 倍的信号窗口, 信噪比高, 而 且使用了可以免洗的钙敏感苂光染料, 更适合进行 $\mathrm{P} 2 \mathrm{X}_{7}$ 受体调节剂的高通量篮选.

\section{参考文献}

1 North R A. Molecular physiology of P2X receptors. Physiol Rev, 2002, 2: 1013-1067

2 North R A. Pharmacology of cloned P2X receptors. Annu Rev Pharmacol Toxicol, 2000, 40: 563-580

3 Davide F, Cinzia P, Elena A, et al. The P2X 7 receptor: A key player in IL-1 processing and release. J Immunol, 2006, 176: 3877-3883

4 Korngreen A, Ma W, Priel Z, et al. Extracellular ATP directly gates a cation-selective channel in rabbit airway ciliated epithelial cells. J Physiol, 1988, 508: 703-720

5 Taylor A L, Schwiebert L M, Smith J J, et al. Epithelial P2X purinergic receptor channel expression and function. J Clin Invest, 1999, 104: $875-884$

6 Baxter A, Bent J, Bowers K, et al. Hit-to-lead studies: The discovery of potent adamantane amide P2X $\mathrm{X}_{7}$ receptor antagonists. Bioorg Med Chem Lett, 2003, 22: 4047-4050

7 Donnelly-Roberts D L, Jarvis M F. Discovery of $\mathrm{P}_{2} \mathrm{X}_{7}$ receptor selective antagonists offers new insights into $\mathrm{P} 2 \mathrm{X}_{7}$ receptor function and indicates a role in chronic pain states. Br J Pharmacol, 2007, 151: 571-580

8 Gever J R, Cockayne D A, Dillon M P, et al. Pharmacology of P2X channels. Pfluge Arch, 2006, 452: 513-537

9 Honore P, Donnelly R D, Namovic M T, et al. A-740003 [N-(1-\{[(cyanoimino)(5-quinoli-nylamino) methyl]amino $\}-2,2-$ dimethylpropyl)2-(3,4-dimethoxyphenyl) acetamide], a novel and selective $\mathrm{P} 2 \mathrm{X}_{7}$ receptor antagonist, dose-dependently reduces neuropathic pain in the rat. $\mathrm{J}$ Pharmacol Exp Ther, 2006, 319: 1376-1385

10 King B F. Novel P2X 7 receptor antagonists ease the pain. Br J Pharmacol, 2007, 151: 565-567

11 Stephen D S, Patrizia D, Pietro G. The P2X 7 purinergic receptor: From physiology to neurological disorders. FASEB J, 2010, 24: 337-345

12 Khakh B S, North R A. P2X receptors as cell-surface ATP sensors in health and disease. Nature, 2006, 442: 527-532

13 Surprenant A, North R A, Buell G, et al. The cytolytic P2Z receptor for extracellular ATP identified as a P2X receptor (P2X ${ }_{7}$ ). Science, 1996, 272: 735-738

14 Chessell I P, Michel A D, Humphrey P P. Properties of the poreforming P2X 7 purinoceptor in mouse NTW8 microglial cells. Br J Pharmacol, 1997, 121: 1429-1437

15 Hickman S E, el Khoury J, Greenberg S, et al. P2Z adenosine triphosphate receptor activity in cultured human monocyte-derived macrophages. Blood, 1994, 84: 2452-2456

16 Zhang J H, Chung T D, Oldenburg K R. A Simple statistical parameter for use in evaluation and validation of high throughput screening assays. J Biomol Screen, 1999, 4: 67-73 